\author{
Rebecca Aslakson \\ Peter Spronk
}

\section{Tasking the tailor to cut the coat: How to optimize individualized ICU-based palliative care?}

Received: 9 October 2015

Accepted: 14 October 2015

Published online: 10 November 2015

(C) Springer-Verlag Berlin Heidelberg and ESICM 2015

\section{R. Aslakson (『)}

Departments of Anesthesiology and Critical Care Medicine and Oncology and Palliative Care Program, The Johns Hopkins School of Medicine, Baltimore, MD, USA

e-mail: raslaks1@jhmi.edu

\section{R. Aslakson}

Department of Health, Behavior and Society, The Johns Hopkins Bloomberg School of Public Health, Baltimore, MD, USA

P. Spronk

Department of Intensive Care Medicine, Gelre Hospitals, Apeldoorn, The Netherlands

e-mail: p.spronk@gelre.nl

Hippocrates said "If we could give every individual the right amount of nourishment and exercise, not too little and not too much, we would have found the safest way to health". Indeed, patients all deserve an individualized approach by caregivers. Palliative care is based on the same principle, as it embraces customized, goal-adaptive care for patients with serious advanced illness, regardless of prognosis or diagnosis. Palliative care emphasizes (1) symptom management with alleviation of physical, psychological, and spiritual distress; (2) improving or maintaining quality of life for patients and their family; (3) compassionate and clear communication, particularly regarding prognosis and patients' goals and values $[1,2]$. Conducted in diverse patient populations, including those with advanced cancer, lung disease, and neurologic disease, as well as frail adults, multiple studies evidence benefits associated with proactive palliative care, including improved quality of life, better symptom management, and higher family satisfaction [2]. For these reasons, palliative care is being integrated throughout the continuum of medical care, including in intensive care units (ICUs) [3, 4]. There are multiple conceptual models for whether palliative care is "integrative" and delivered by the ICU team, "consultative" and delivered by a specialist palliative care team, or "mixed" and delivered by some combination of the two [5-7] (Table 1). Previous studies suggest that both consultative and integrative models of ICU-based care can be effective [4], and the "ideal" model for any ICU will likely vary by hospital and ICU resources, patient populations, and local culture. A variety of ICU-based palliative care delivery models have been evaluated $[4,8,9]$, and yet more data on the effectiveness of different models are needed for individual ICUs to feasibly choose the delivery modality that is "right" for their unique circumstances.

In a recent article in Intensive Care Medicine, Braus et al. [10] describe a single-center, before-and-after study at an academic, tertiary care hospital in Wisconsin, USA. The intervention consisted of a palliative care clinician, primarily a clinical nurse specialist, rounding with the ICU team for patients who met trigger criteria for "high risk of mortality, morbidity, or unmet palliative care needs". The palliative care clinician reviewed the medical records of patients triggered by the above criteria and then notified the ICU team that a patient met trigger criteria. On subsequent days during rounds, the palliative care clinician could make suggestions attempting to "nudge" the ICU team to better integrative palliative care, including discussions of prognosis and proactive family meetings. The primary outcome was the proportion of patients who had an interdisciplinary family meeting in the ICU, with secondary outcomes including time between ICU admission and a family meeting, ICU and 
Table 1 Conceptual models for palliative care delivery [5-7]

\begin{tabular}{|c|c|}
\hline Model type & Description \\
\hline "Primary" "generalist", or & $\begin{array}{l}\text { Palliative care delivered by the primary or ICU team, without involvement of palliative care specialists, } \\
\text { and typically involving basic management of symptoms and communication regarding prognosis }\end{array}$ \\
\hline "Specialty" or "consultative" & $\begin{array}{l}\text { Palliative care delivered by palliative care specialists and typically involving management of refractory } \\
\text { and/or more complex symptoms with proactive conflict resolution and nuanced discussions regarding } \\
\text { potentially non-beneficial treatments }\end{array}$ \\
\hline "Mixed" & $\begin{array}{l}\text { A combination approach where basic symptom management and communication is managed by the } \\
\text { primary or ICU team with consultation of a specialist team, as needed, to address more complex } \\
\text { symptomatology and/or communication scenarios }\end{array}$ \\
\hline
\end{tabular}

ICU Intensive care unit

hospital length of stay, ICU and in-hospital mortality, family satisfaction, family member symptoms of depression and PTSD, and family assessed quality-of-death for ICU decedents. No sample size calculations were performed a priori, and the study was underpowered to detect differences for many of these outcomes.

There were 100 patients enrolled in the "pre" intervention phase and 103 patients enrolled during the "post" intervention phase. The intervention was associated with increased documentation of an ICU family meeting during the ICU stay [relative risk (RR) 1.63, $95 \%$ confidence interval (CI) 1.14-2.07] and a $41 \%$ shortening of time between ICU admission and a family meeting (95\% CI 52-28\% shorter). Although after adjustment for confounders, the ICU length-of-stay (LOS) was not different, the hospital LOS significantly decreased (median LOS from 13 to 11 days with adjusted model; $p<0.001$ ). Among decedents and after adjusting for confounders, both ICU and hospital length of stay decreased (19\% reduction, $p=0.043 ; 30 \%$ reduction, $p<0.001$, respectively). There were no statistically significant differences for ICU mortality, hospital mortality, psychological outcomes, or satisfaction with care.

In the context of past ICU-based palliative care trials, there are several unique aspects of the approach chosen by Braus et al. [10]. Unlike traditional consultative ICU-based palliative care interventions where either patients meeting triggers receive a full palliative care consultation $[11,12]$ or a palliative care-related "navigator" interacts with both the ICU clinician team and ICU patients and their family members [13], Braus and colleagues instead tested a model where the palliative care clinician interacts only with the ICU clinicians, with a goal of supporting primary palliative care provided by that ICU team. Also unlike traditional integrative ICU-based palliative care interventions [4], the model tested by these authors does not require further training or extra duties to the ICU team; rather, the palliative care clinician accompanies the team on rounds, but it is ultimately the decision of the ICU team on whether-and if so, when - to convene a family meeting or to address a palliative care-related issue.

Many previous ICU-based palliative care interventions decreased ICU or hospital LOS [4] and thus it is perhaps not surprising that this intervention also, at least partially, was associated with changes in resource utilization. Indeed, perhaps it does not take a strong "nudge" for ICU clinicians to prioritize family meetings for their sickest patients; the Braus model seems relatively simple to implement and elegantly incorporates palliative care in the "normal" daily ICU routine, rather than setting palliative care aside as something requiring a separate consultation.

Yet, also consistent with many past studies [4], this approach was not associated with improvement in familyreported outcomes. While one large trial of an integrative ICU-based palliative care approach studied in 22 French ICUs was associated with significant decreases in family member anxiety, depression, and post-traumatic stress disorder levels [14], only a handful of ICU-based palliative care studies [15-17] have measured family-reported outcomes other than "family satisfaction". Moreover, ICU-based palliative care trials have thus far not measured truly patient-centered outcomes, such as symptom scores or quality of life [18]. Furthermore, other than the previously mentioned French trial [14], all ICU-based palliative care trials have been completed in North America. There is a dearth of adequately powered studies concerning both the impact of ICU-based palliative care on patient- and family-centered outcomes and the delivery of palliative care in non-North American critical care settings.

Much as a tailor carefully fashions a garment to be both responsive to community fashion as well as attuned to the wearer's unique frame and countenance, so ICU clinicians and hospitals must choose ICU-based palliative care approaches that are conducive to their culture as well as attentive to the unique needs of their patients and clinicians. As one size definitely will not "fit all", different approaches to ICU-based palliative care-including approaches tailored to European ICUs - need to be both developed and tested. We all want our ICU patients, particularly those who are the sickest and most vulnerable-as well as their families-to receive timely and compassionate palliative care and communication that is both sensitive to their unique needs as well as responsive to the often-volatile clinical circumstances. Moreover, to 
evaluate effectiveness of ICU-based palliative care, we also must develop or embrace multi-faceted, innovative, valid, and patient- and family-centered metrics. Only then will we be able to implement evidence-based approaches and evaluate the impact of our efforts to deliver tailored palliative care to the right patients, in the right way, and at the right time.

\section{Compliance with ethical standards}

Conflicts of interest On behalf of both authors, the corresponding author states that there is no conflict of interest.

\section{References}

1. National Consensus Project (2013)

National consensus for quality palliative care: clinical practice guidelines for quality palliative care, 3rd edn. Available at:

http://

www.nationalconsensusproject.org.

Accessed 29 Sep 2015

2. Kelley AS, Morrison RS (2015)

Palliative care for the seriously ill. N Eng J Med 373:747-755

3. Aslakson RA, Curtis JR, Nelson JE (2014) Concise definitive review: the changing role of palliative care in the ICU. Crit Care Med 42:2418-2428

4. Aslakson RA, Cheng J, Vollenweider D et al (2014) Evidence-based palliative care in the intensive care unit: a systematic review of interventions. J Pall Med 17(2):219-235

5. Institute of Medicine (2014) Dying in America: improving quality and honoring individual preferences near the end of life. National Academies, Washington, DC

6. Quill TE, Abernethy AP (2013) Generalist plus specialist palliative care-creating a more sustainable model. N Eng J Med 368(13):1173-1175

7. Nelson JE, Bassett R, Boss RD et al (2010) Models for structuring a clinical initiative to enhance palliative care in the intensive care unit: a report from the IPAL-ICU project (improving palliative care in the ICU). Crit Care Med 38:1765-1772
8. Khandelwal N, Kross EK, Engelberg RA et al (2015) Estimating the effect of palliative care interventions and advance care planning utilization: a systematic review. Crit Care Med 43:1101-1111

9. Scheunemann LP, McDevitt M, Carson SS et al (2011) Randomized, controlled trials of interventions to improve communication in intensive care: a systematic review. Chest 139(3):543-554

10. Braus N, Campbell TC, Kwekkeboom KL et al (2015) Prospective study of a proactive palliative care rounding intervention in a medical ICU. Intensive Care Med. doi: 10.1007/s00134-015-4098-1

11. Campbell ML, Guzman JA (2003) Impact of a proactive approach to improve end-of-life care in a medical ICU. Chest 123(1):266-271

12. Campbell ML, Guzman JA (2004) A proactive approach to improve end-oflife care in a medical intensive care unit for patients with terminal dementia. Crit Care Med 32(9):1839-1843

13. Curtis JR, Treece PD, Nielsen EL, et al. (2015) Randomized trial of communication facilitators to reduce family distress and intensity of end-oflife care. Am J Respir Crit Care Med. doi: 10.1164/rccm.201505-0900OC
14. Lautrette A, Darmon M, Megarbane B et al (2007) A communication strategy and brochure for relatives of patients dying in the ICU. N Eng $\mathrm{J}$ Med 356:469-478

15. Curtis JR, Curtis JR, Nielsen EL, Treece PD et al (2011) Effect of a quality-improvement intervention on end-of-life care in the intensive care unit: a randomized trial. Am J Respir Crit Care Med 183(3):348-355

16. Curtis JR, Treece PD, Nielsen EL, Downey L, Shannon SE, Braungardt T, Owens D, Steinberg KP, Engelberg RA (2008) Integrating palliative and critical care: evaluation of a qualityimprovement intervention. Am J Respir Crit Care Med 178(3):269-275

17. Puntillo K, Nelson JE, Weissman D, Curtis R, Weiss S, Frontera J, Gabriel M, Hays R, Lustbader D, Mosenthal A, Mulkerin C, Ray D, Bassett R, Boss R, Brasel K, Campbell M, Advisory Board of the Improving Palliative Care in the ICU (IPAL-ICU) Project (2014) Palliative care in the ICU: relief of pain, dyspnea, and thirst - a report from the IPAL-ICU Advisory Board. Intensive Care Med 40(2):235-248

18. Aslakson RA, Bridges JFP (2013) Assessing the impact of palliative care in the intensive care unit through the lens of patient-centered outcomes research. Curr Opin Crit Care 19:504-510 